\title{
A preliminary report on stem cell therapy for neuropathic pain in humans
}

This article was published in the following Dove Press journal:

Journal of Pain Research

8 May 2014

Number of times this article has been viewed

\author{
E Russell Vickers' \\ Elisabeth Karsten ${ }^{2}$ \\ John Flood ${ }^{3}$ \\ Richard Lilischkis ${ }^{2}$ \\ 'Sydney Oral and Maxillofacial \\ Surgery, NSW, Australia; ${ }^{2}$ Regeneus \\ Ltd, Gordon, NSW, Australia; \\ ${ }^{3}$ St Vincents Hospital, Sydney, NSW, \\ Australia
}

Correspondence: E Russell Vickers Sydney Oral and Maxillofacial Surgery, Suite I40I, 520 Oxford St, Bondi Junction, NSW, Australia $\mathrm{Tel}+61293692153$

Fax +6I 293692154

Email oralmaxima@bigpond.com
Objective: Mesenchymal stem cells (MSCs) have been shown in animal models to attenuate chronic neuropathic pain. This preliminary study investigated if: i) injections of autologous MSCs can reduce human neuropathic pain and ii) evaluate the safety of the procedure.

Methods: Ten subjects with symptoms of neuropathic trigeminal pain underwent liposuction. The lipoaspirate was digested with collagenase and washed with saline three times. Following centrifugation, the stromal vascular fraction was resuspended in saline, and then transferred to syringes for local injections into the pain fields. Outcome measures at 6 months assessed reduction in: i) pain intensity measured by standard numerical rating scale from $0-10$ and ii) daily dosage requirements of antineuropathic pain medication.

Results: Subjects were all female (mean age 55.3 years \pm standard deviation [SD] 14.67; range 27-80 years) with pain symptoms lasting from 4 months to 6 years and 5 months. Lipoaspirate collection ranged from $102-214 \mathrm{~g}$ with total cell numbers injected from 33 million to 162 million cells. Cell viability was $62 \%-91 \%$. There were no systemic or local tissue side effects from the stem cell therapy ( $\mathrm{n}=41$ oral and facial injection sites). Clinical pain outcomes showed that at 6 months, $5 / 9$ subjects had reduced both pain intensity scores and use of antineuropathic medication. The mean pain score pre-treatment was 7.5 (SD 1.58) and at 6 months had decreased to 4.3 (SD 3.28), $P=0.018$, Wilcoxon signed-rank test. Antineuropathic pain medication use showed $5 / 9$ subjects reduced their need for medication (gabapentin, $P=0.053$, Student's $t$-test).

Conclusion: This preliminary open-labeled study showed autologous administration of stem cells for neuropathic trigeminal pain significantly reduced pain intensity at 6 months and is a safe and well tolerated intervention.

Keywords: adipose, stem cells, neuropathic, orofacial, trigeminal

\section{Introduction}

Neuropathic pain is one of the most difficult chronic pain conditions to treat and affects approximately $6 \%$ of the population and can be caused by any number of events. ${ }^{1,2}$ Word descriptor qualities of neuropathic pain that patients report are typically sharp, shooting, burning, electric, or lancinating. ${ }^{3,4}$ The pathogenesis involves molecular changes (increased sodium channel density, expression of substance $\mathrm{P}$ and nerve growth factor), and cellular changes (neuroplasticity and central sensitization). ${ }^{5}$ Central sensitization provides an explanation for the dimensional increase of the pain field and temporal qualities observed in chronic pain patients. It is also well-established that the combined action of glial cells and cytokine modulated inflammation plays an important role in the development and progression of neuropathic pain. ${ }^{6-8}$ Neuropathic pain typically presents in the clinic with hyperalgesia, allodynia, spontaneous ectopic 
discharges, secondary activation, and maintenance of sympathetically maintained pain and regional myofascial pain. ${ }^{9,10}$ Neuropathic trigeminal pain comprises several diagnostic states, including maxillofacial neuropathic pain from trauma, burning mouth syndrome, and atypical odontalgia (phantom tooth pain). Considering trigeminal neuropathy is a relatively localized form of neuropathic pain, patients with this pain state are considered an ideal group for the investigation of progressive new therapies.

Neuropathic pain responds poorly to opioid and overthe-counter analgesics. First-line antineuropathic medication utilizes tricyclic antidepressants and anticonvulsants administered to approximately $40 \%$ of people with facial neuropathies. ${ }^{2}$ However, pain relief experienced with these treatments is only moderate. Sindrup and Jensen ${ }^{11}$ identified that, on average, a minimum of three peripheral and central chronic pain patients need to be treated before one patient will experience a $50 \%$ improvement in pain symptoms. In addition, approximately $25 \%$ of people with facial neuropathies receive no treatment at all. ${ }^{2}$ Compliance with antineuropathic medication can be problematic for patients with potential side effects of weight gain, drowsiness, dry mouth, negative mood changes, and increased suicide risk.

The use of mesenchymal stem cells (MSCs) as a therapeutic agent is a growing field due to the reported safety in transplantation and efficacy in a range of conditions. Mazzini et $\mathrm{al}^{12}$ demonstrated the safety of MSCs for up to 9 years after intravenous administration into amyotrophic lateral sclerosis patients, and no adverse events were observed in a human stem cell clinical trial of 53 participants. ${ }^{13}$ Adipose tissue has been identified as an advantageous source of MSCs over traditional sources such as the bone marrow, with MSCs present at significantly higher concentrations from lipoaspirate. ${ }^{14}$ The stromal vascular fraction (SVF) of adipose tissue is comprised of a mixed population of cells including MSCs, red blood cells, leukocytes, and endothelial cells. The administration of the whole SVF for the treatment of disease has a range of benefits. It subtracts the need to expand the MSCs in vitro, and as a result, autologous treatments can be performed on the same day that the liposuction occurs. It has also been demonstrated that cell signaling and the cytokine profile are enhanced when the SVF is co-cultured with adipocytes, which results from the cross-talk between cells in close proximity to each other. ${ }^{15}$ SVF has undergone preliminary investigations for human clinical conditions and experimental animal models. Riordan et $\mathrm{l}^{16}{ }^{1}$ treated three multiple sclerosis patients with autologous SVF and additional injections of allogeneic CD34+ cells and MSCs with positive results. Direct implantation of the SVF into in vivo animal models and in vitro culture models has stimulated neovascularization and reduced inflammatory symptoms. ${ }^{17,18}$

The use of MSCs in the treatment of neuropathic pain is a rapidly expanding field due to a few core properties of these cells. MSCs have been reported to exert an antiinflammatory effect through cytokine release that may combat the pathological inflammation involved in neuropathic pain, ${ }^{18,19}$ and have been shown to play an important role in nerve healing and regeneration. ${ }^{20}$ This hypothesis has been tested in animal models of trigeminal neuropathic pain, diabetic neuropathy, and hind paw neuropathic pain, all of which showed promising results, with a significant reduction in neuropathic pain symptoms. ${ }^{21-23}$ Sacerdote et $\mathrm{al}^{24}$ reported that the use of adipose-derived MSCs in an animal model of hind paw neuropathic pain resulted in a decrease in the proinflammatory cytokine interleukin (IL)- $1 \beta$ and an increase in the anti-inflammatory cytokine IL-10 in the lesioned nerve, as well as a significant reduction in thermal hyperalgesia and mechanical allodynia.

In this preliminary study, we investigated the safety and efficacy of injecting the SVF-containing adipose-derived stem cells in ten subjects with an established diagnosis and who demonstrated neuropathic pain symptoms. The rationale for injecting MSCs directly into the pain sites was based on two animal studies comparing high/low dose local administration of MSCs to alleviate neuropathic pain. ${ }^{21,22}$ Both studies demonstrated superior efficacy of the higher concentration of cells. Thus, the purpose of this investigation was to assess proof of concept and evaluate the safety of a high concentration of MSCs in patients with clinical neuropathic pain. The literature to date is very limited for evaluating the therapeutic role of MSCs in treating neuropathic pain in humans. Only one case study has been published and it reported a good response from expanded umbilical cord MSCs injected intrathecally for neuropathic pain. ${ }^{25}$ In contrast, a casecontrolled study of intrathecally administered bone marrowderived MSCs for human spinal cord injury showed a high rate of inducing neurogenic inflammation, although causative mechanisms could not be identified. ${ }^{26}$ The authors, to their knowledge, believe that this is the first reported study of administering stem cells in the peripheral nervous system for the treatment of human neuropathic pain.

\section{Materials and methods}

This study was conducted on adult, private-consenting patients as permitted in Australia under the Australian Government legislative framework administered and regulated by the 
Therapeutic Goods Administration (Therapeutic Goods Order No 1 of 2011, the Excluded Goods Order) for autologous stem cell procedures. Specifically, this legislature allows medically unregulated products derived from human tissue to be used under the management of a registered medical practitioner, and that the procedure occurs within a single treatment. In addition, regulations and guidelines set by the Australian Health Practitioner Regulation Agency (AHPRA) for clinicians to perform treatments include relevant scientific evidence, explanation, and details of the treatment procedure and potential risks. The purpose of this exemption allows for preliminary studies to be conducted in order that subsequent research funding and university hospital ethical committees have published, human-based evidence prior to submission for the approval of double-blind, randomized controlled trials (RCTs). Based on these Australian Government regulations, adult subjects were recruited from the Sydney Oral and Maxillofacial Surgery Pain Clinic, a private clinic with no direct university or hospital affiliation. ERV is the director of the clinic and is an AHPRA-registered specialist. In this study, subjects were being managed on standard anti-neuropathic medication, typically amitriptyline and gabapentin. Informed consent to administer autologous stem cell therapy was obtained from all patients. Subjects stated that their reasons for considering stem cell intervention were inadequate pain relief from the medication or excessive side effects to the therapeutic level of the drugs. Stem cell processing was conducted at no cost for the subjects. No financial remuneration was provided to subjects for the procedure. Data were collected from each patient for age, sex, pain intensity, medication type and dose for pain management, etiology, and duration of pain (Table 1).

Clinical and radiographic examination was conducted on all subjects to exclude nociceptive pathology such as dental caries, occlusion and bite problems, periodontal disease, jaw joint disk disorder, or arthritis and other differential diagnoses (ie, migraine, cervicogenic pain). Subjects underwent a general physical and medical assessment. Patients were screened by a questionnaire prior to treatment and were to be excluded if positive for hepatitis B and C and HIV. Blood screens were not routinely conducted as the test procedure was for local administration of MSCs that were not expected to influence circulating white blood cell count.

Patients were treated at the North Shore Specialist Day Hospital (Greenwich, NSW, Australia). Acquisition of lipoaspirate from the bilateral lumbar region was performed by author JF. The clinical procedure was administration of local anesthetic prior to two small lumbar incisions and then adipose tissue collected by tumescent liposuction. Approximately 100-200 g of tissue was harvested by tumescent liposuction into sterile, sealed containers. Stem cell processing was conducted by Regeneus Ltd (Gordon, NSW, Australia). The SVF was isolated through collagenase digestion of lipoaspirate, as previously reported. ${ }^{15}$ Briefly, lipoaspirate was digested for 20 minutes with $0.5 \mathrm{mg} \mathrm{mL}^{-1}$ of human grade collagenase (NB6 GMP; SERVA Electrophoresis GmbH, Heidelberg, Germany). Subsequently, the digested material was washed and centrifuged $(1,500 \mathrm{~g}, 5$ minutes) three times in sterile, saline-containing vancomycin $\left(0.05 \mathrm{mg} \mathrm{mL}^{-1}\right)$. After washing, the SVF and part of the adipocyte layer was resuspended in sterile, saline-containing vancomycin $\left(0.05 \mathrm{mg} \mathrm{mL}^{-1}\right)$. Aliquots of the reconstituted SVF pellet were prepared in $1 \mathrm{~mL}$ and $2 \mathrm{~mL}$ syringes, with a 25 -gauge needle for injection. The injection sites for the SVF were perineural, directly into the center or origin of pain, and the adjacent pain field of the affected branches of the trigeminal nerve. To reduce pain from the injection of the SVF for the subject, the pain field was pretreated with EMLA cream 5\% topical anesthetic (AstraZeneca, North Ryde, Australia) for 3 minutes, then 1-2 mL of $1 \%$ lidocaine (without vasoconstrictor). The procedure was a single-stage medical procedure lasting 2-3 hours in total in a hospital theatre. The procedural sequence was liposuction (45 minutes), followed by laboratory lipoaspirate processing (90 minutes), and SVF injections (10 minutes).

The primary treatment outcome to be measured was change in pain intensity and the secondary outcome measure was any reduction in the daily consumption of antineuropathic medication. Subjects were reviewed for safety evaluation for any clinical sign of trigeminal nerve deficit (paresthesia, dysesthesia), facial nerve paresis, infection, and unusual swellings or lesions at the SVF injection sites by ERV (specialist oral and maxillofacial surgeon). Review appointments were made for subjects as close as possible, at 1 week, and 1, 3, and 6 months, after the procedure for pain scores and clinical assessment of the injection sites. Data and statistical analysis were reported on all subjects at the 6-month outcome interval.

All values were reported as mean \pm standard deviation (SD). Differences in pain intensity scores were statistically evaluated with the Wilcoxon signed-rank test and differences in medication assessed with a paired, two-tailed, Student's $t$-test with significance levels at $P<0.05$.

\section{Results}

Ten subjects (all females) were recruited for the procedure with a mean age of 55.3 years \pm SD 14.67 (range 27-80 years). 


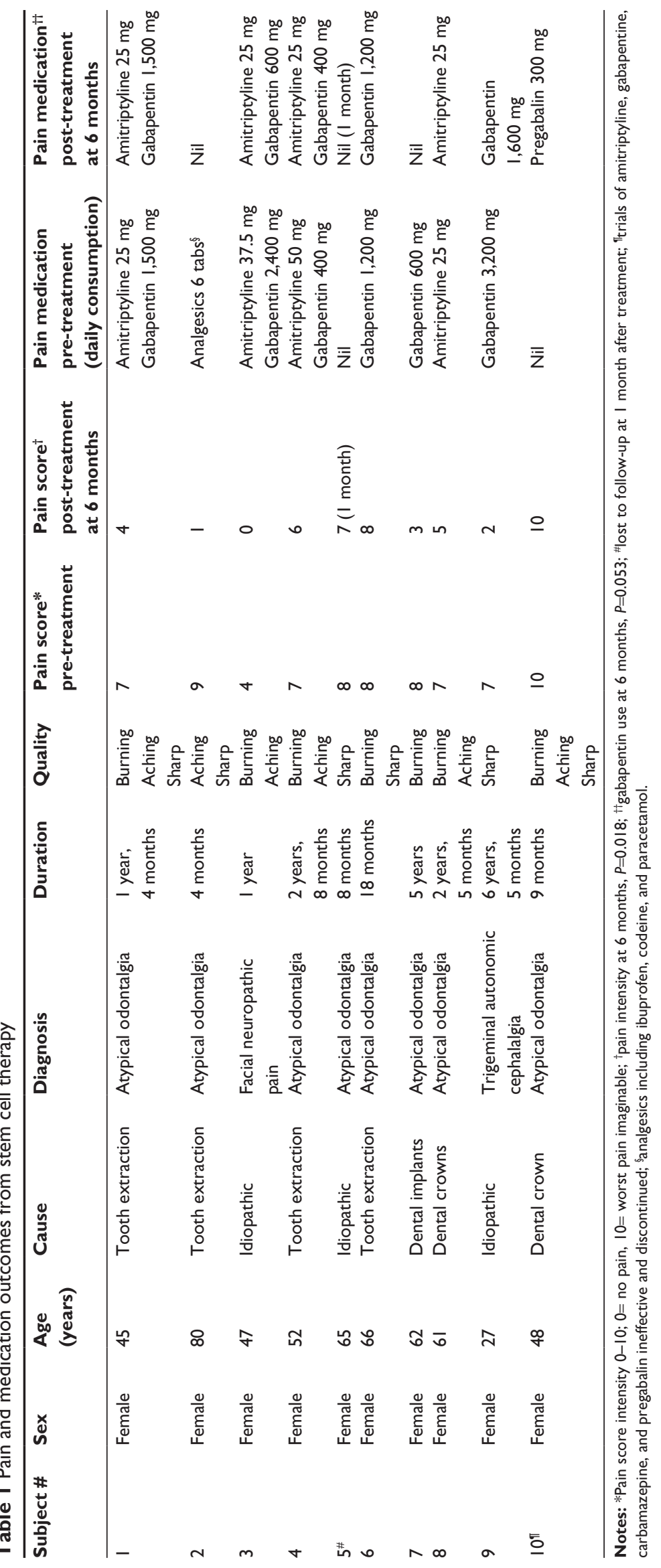


Eight subjects had a diagnosis of atypical odontalgia (intra-oral neuropathic pain), one patient with facial neuropathic pain, and one patient with a diagnosis of trigeminal autonomic cephalalgia (SUNCT [Short-lasting Unilateral Neuralgiform headache with Conjunctival injection and Tearing]) with facial neuropathic pain symptoms. No subject showed any side effects of the treatment. There was no evidence of unusual localized swellings or lesions at any of the injection sites ( $n=36$ intraoral sites and $n=5$ facial sites) nor evidence of general or medical health issues at any follow-up stage (to 6 months). Table 1 shows a summary of demographic data, cause of pain, change in pain intensity scores, and change in required medication for adequate pain relief. One subject was lost to follow-up after 1 month post-review. Laboratory results on adipose tissue collection, total cell numbers, and viability are shown in Table 2.

The neuropathic pain group was found to be a heterogeneous group when listing their pre-treatment pain word qualities; six patients reported sharp neuralgic pain, seven patients with burning pain, and six with aching pain. The mean pain score (numerical rating scale, $0=$ no pain, $10=$ worst pain imaginable) for the group prior to stem cell treatment was 7.5 (SD 1.58) and had decreased at 6 months to 4.3 (SD 3.28; $P=0.018$, Wilcoxon signed-rank test; Figure 1). The results showed a reduction in pain intensity scores from the stem cell treatment in 7/9 patients (two with marginal improvement and five subjects with good-to-excellent pain reduction). Five of these positive responders also reduced their need for gabapentin medication (gabapentin, $P=0.053$, Student's $t$-test; amitriptyline, $P=0.22$; Figure 2), three required the same level of medication, and one subject commenced multiple medication trials within the 6-month period without any pain relief, with all medication subsequently discontinued. This subject (\#10) with a pain intensity of 10/10 underwent trials of amitriptyline, carbamazepine, gabapentin, and pregabalin in addition to the stem cells over 6 months. Subjects \#6 and \#10, while reporting no reduction for pain intensity on the numerical scale, surprisingly did report that their pain had decreased from "severe to moderate" on a separate word descriptor scale.

\section{Discussion}

The stem cell study showed a typical response from a general pain population involved in a trial with loss of one patient to follow-up, despite repeated phone calls and occasional non-compliance of review appointments in others. The therapeutic action of stem cells were typical of a pharmacological intervention, with two non-responders and others with varying degrees of mild, moderate, and exceptional pain reduction. The majority of subjects, however, reported a positive effect from stem cell therapy with pain reduction in addition to lowering antineuropathic medication dose to enable an improved quality of life with fewer drug side effects. Two subjects had excellent responses. This was an open-label trial utilizing an injection/surgical approach to treatment, and likely to have a high placebo response. Typically, placebo-controlled trials for neuropathic pain medications such as gabapentin are analyzed for a maximum of 12 weeks to observe the effect (if any) of the treatment. ${ }^{27-29}$ This study is likely to have an increased placebo effect due to the injection/surgical approach to treatment. ${ }^{30-32}$ For this reason, the review period post-treatment was extended to 26 weeks (Figure 1) to lessen the placebo effect and to support the concept of a true biological improvement from the procedure. Multiple pain data points obtained over the 6-month period showed a steady and gradual reduction in

Table 2 Total numbers of cells and cell viability

\begin{tabular}{|c|c|c|c|c|c|c|}
\hline Subject \# & Total cells & Fat (g) & Total cells/g & Viability (\%) & $\begin{array}{l}\text { Number of } \\
\text { injection sites }\end{array}$ & $\begin{array}{l}\text { Total volume } \\
\text { injected ( } \mathrm{mL})\end{array}$ \\
\hline I & $162,337,963$ & 150 & $\mathrm{I}, 082,253$ & 91 & 3 & 6 \\
\hline 2 & $40,110,519$ & 160 & 250,691 & 66 & 4 & 8 \\
\hline 3 & $68,683,78 I$ & 170 & 404,022 & 82 & 4 & 6 \\
\hline 4 & $126,090,064$ & 214 & 589,206 & NA & 5 & 8 \\
\hline 5 & $77,937,200$ & 140 & 556,694 & NA & 5 & 8 \\
\hline 6 & $99,621,844$ & NA & NA & 81 & 4 & 8 \\
\hline 7 & $32,904,794$ & 102 & 322,596 & 62 & 4 & 8 \\
\hline 8 & $43,391,643$ & 120 & 361,597 & 79 & 3 & 4 \\
\hline 9 & $76,641,789$ & 127 & 603,479 & NA & 5 & 8 \\
\hline 10 & $44,905,058$ & 116 & 387,113 & 70 & 4 & 8 \\
\hline
\end{tabular}

Notes: Cell per gram represents total cells per gram (viable and non-viable cells); NA = values not available on three patients due to technical equipment problems on same day of procedure; injection sites for subject \#9 were facial injections, all other subjects were intraoral injections; total volume injected was mL of normal saline containing total cell suspension. 


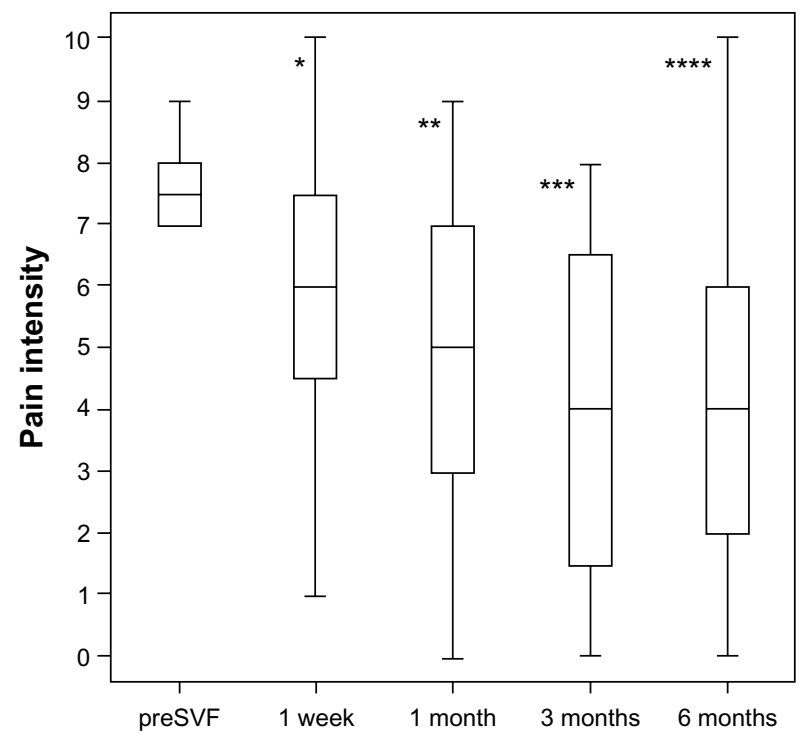

Figure I Pain intensity scores (median and quartiles) in ten subjects with neuropathic trigeminal pain over 6 months following autologous adipose stem cell injection.

Notes: Subjects completed pain scores at the pre-treatment SVF stage $(n=10$ scores), I week after treatment ( $n=7$ scores), I month $(n=6), 3$ months $(n=7)$, and at 6 months $(n=9)$. Pain reduction was significant at all time points at I week $(P=0.04 I)^{*}$, I month $(P=0.026)^{* *}, 3$ months $(P=0.028)^{* * * *}$ and 6 months $(P=0.018)^{* * * *}$ (Wilcoxon signed-rank test) compared with pre-treatment pain.

Abbreviation: SVF, stromal vascular fraction.

pain, suggesting actual progressive biological changes. The orofacial region is considered an ideal site for clinical experimentation of analgesic agents in pain research; in particular, the impacted bilateral third molar model of the test agent versus placebo (or current standard agent) for acute pain. Similarly, bilateral trigeminal neuropathic pain would conceivably be an ideal model for other new treatments such as cell-based therapies.

There was no patient reporting "miraculous" pain relief directly after the procedure, reducing the likelihood of a major placebo response. Moreover, the neuropathic group had a history of multiple drug trials to identify individualized polypharmacy pain management. This group of patients is not naïve to procedures and is likely to be less affected by the placebo. The two non-responders over the 6-month trial (subject \#6 with 8/10, and subject \#10 with 10/10 intensity) both had high levels of background personal stress that were pre-existing and continued throughout the 6-month evaluation. Subject \#6 had a confounding clinical representation with "severe pain" documented in the clinical notes at the pre-treatment stage that subsequently reduced to "moderate pain" but still had an intensity of $8 / 10$. Subject \#10 reported pain of $8 / 10$ at 3 months but returned to $10 / 10$ at 6 months. In both cases, prior to stem cell treatment, they would contact the clinic frequently with several phone calls each week or frequent appointments to report extreme pain. However, in the 6 months post-stem cells this did not occur. In the clinical setting, there was less distress, suggesting less pain intensity despite unchanged pain intensity scores at 6 months in the outcome-measuring instrument.

One intriguing result was seen in the youngest patient (27 years) who had clearly visible signs of autonomic dysfunction of red swollen ankles and feet that completely cleared. In addition, this subject had received multiple botox injections into the facial region by a regional hospital pain clinic. While initially helpful in reducing pain, the botox injections became ineffective and caused significant facial tissue loss. The stem cells completely recovered the tissue loss with restoration of normal facial contour and esthetics. The results from this subject showed adipose stem cells to be an attractive cellular therapy for cosmetic applications. Somewhat surprisingly, one of the best responders was the oldest patient at 80 years, indicating stem cells are still biologically potent at an advanced age with the potential for investigating older age diseases such as diabetic neuropathy. Stolzing et al, ${ }^{33}$ however, have reported the contrary and demonstrated that the "fitness" of bone-marrow-derived MSCs do appear to decline with donor age. The disparities may be a result of a few factors, one being the source of the MSCs (adipose tissue versus bone marrow), and another being the theorized action of the stem cells. It is commonly accepted that MSCs secrete a range of factors to achieve their therapeutic effect and so it is possible that the secretion profile of the cells remains unaffected by age. ${ }^{15,17,34}$ Stolzing et $\mathrm{al}^{33}$ did not test for any changes in the secretion profile of cells, but instead focused on the presence of cell markers, the differentiative ability, and growth dynamics.

The safety of autologous MSC administration as a cellular therapy is well-established and accepted in both human and animal trials. ${ }^{12,13,35}$ Safety was evaluated in this case series with close clinical inspection at several time points throughout the trial. There were no visible deleterious changes at any of the injection sites. In addition, there were no changes to normal nerve physiology of the involved cranial nerves where stem cells were administered; specifically, no numbness, tingling, dysesthesia on the lower lip, chin, lateral border of the tongue or face (trigeminal nerve branches V2 and V3), and no report of motor nerve dysfunction to the face ( $\mathrm{C}$, facial nerve) or jaw (motor branch of the trigeminal nerve). In addition, no infection of the environment was observed where the stem cells were injected. The oral cavity contains a vast array of commensal microflora, estimated to have over 800 species of bacteria, viruses, and fungal elements that would have seeded the stem cells on injection, 

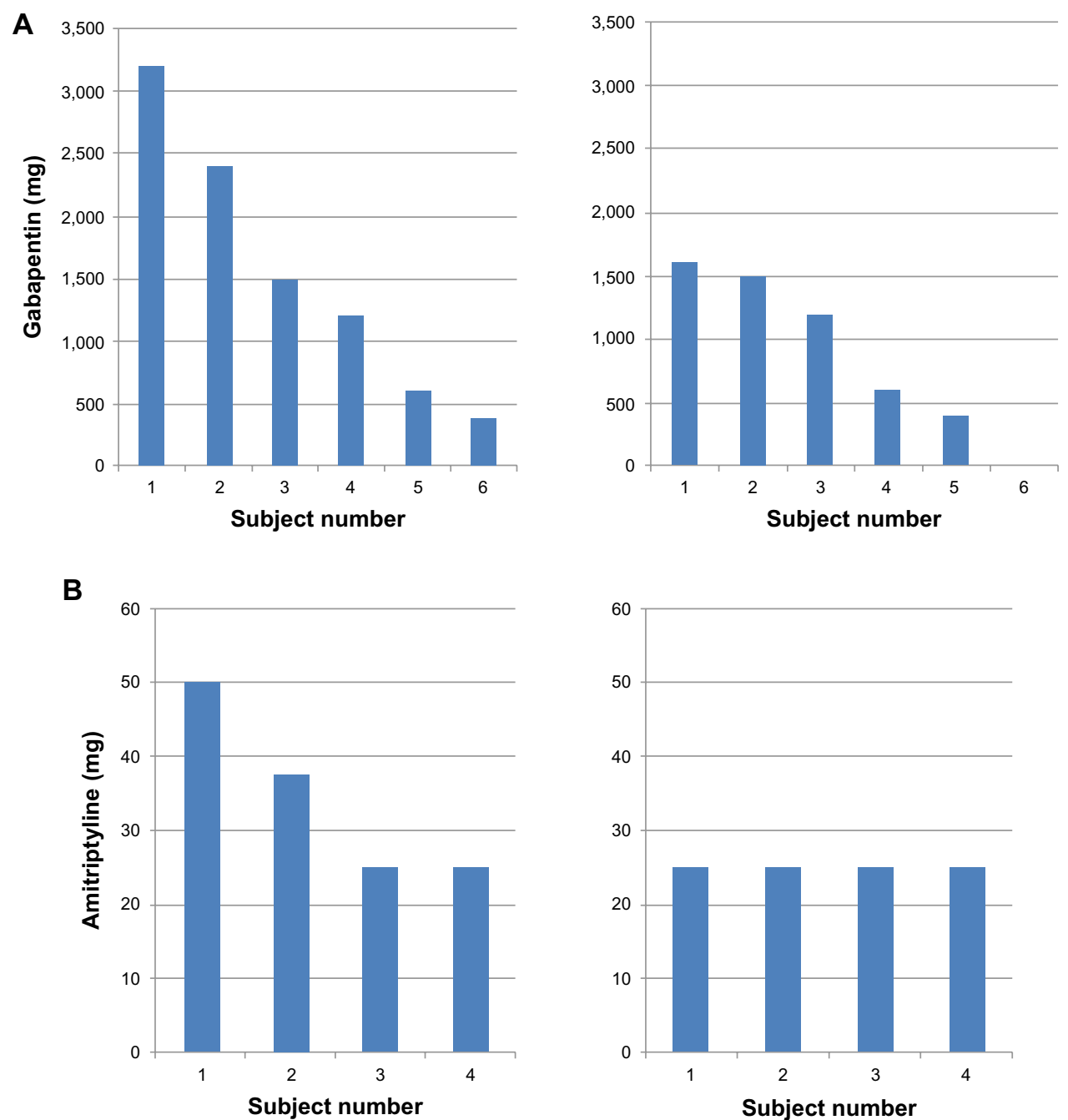

Figure 2 Change in daily dosage requirements of medication.

Notes: (A) gabapentin in the group from pre-treatment stage to completion of the trial at 6 months. At the pre-SVF data point, there were six subjects using gabapentin (mean =I,490 mg; SD I, I60.26; range 600-3,200 mg; total 9,300 mg) and at 6 months, there was a trend for lower analgesic need for gabapentin - one subject discontinued the drug and daily drug consumption reduced in the group (mean $=823 \mathrm{mg}$; SD 7|3.35; range 400-I,600 mg; total 5,300 mg) $(P=0.053$, Student's $t$-test); (B) change in total daily dosage of amitriptyline in the group from pre-treatment stage to completion of the trial at 6 months. At the pre-SVF data point, there were four subjects using amitriptyline (mean =37 mg; SD II.97; range 25-50 mg; total $137.5 \mathrm{mg}$ ) and at 6 months, all four subjects continued using the drug (mean =25 mg; SD 0; total $100 \mathrm{mg}$ ) $(P=0.22$, Student's $t$-test).

Abbreviations: SD, standard deviation; SVF, stromal vascular fraction.

but no evidence of infection was evident. In essence, there appeared to be minimal risk of postoperative infection in the orofacial region from stem cell therapy. This is similar to the minimal risk of intraoral dental local anesthetic injections by dentists in a "contaminated" oral environment, and minimal risk from cosmetic injections in the face. All subjects reported the stem cell procedure to be relatively simple and well tolerated, and the treatment protocol for administering the cells was analogous to their previous familiar exposure of routine dental injections.

Neuropathic pain is recognized as a difficult and challenging pain state to obtain meaningful and prolonged pain reduction. Prior to the trial, the subjects were undergoing pain management for months to years on appropriate antineuropathic medication at therapeutic dose ranges. Despite utilizing proven pharmacotherapy, the mean pain intensity of 7.5 still reveals a major deficiency in treating chronic neuropathic pain to achieve an acceptable quality of life. This trial showed a good response to a single dose of SVF. As previously mentioned, the SVF contains a heterogeneous population of cells and absolute assignment of therapeutic effect can not yet be attributed solely to the MSCs. For this study, the typical cell distribution was $45 \% \pm 13.7 \%$ CD90 positive cells, $16.3 \% \pm 7.6 \%$ CD31 positive cells, and $45 \% \pm 14.4 \% \mathrm{CD} 45$ positive cells. Although there is an overlap in markers (endothelial cells are both CD31 and CD90 
positive), CD90 is one of the recognized markers for MSCs. This data is currently under review by Karsten et al. Further studies are warranted for multiple dosage strategies such as administration of second and third injections at 3 and 6 months, respectively. Considering that storage of stem cells is becoming increasingly accessible with commercial stem cell banking facilities, further benefit from stem cell therapy is likely in chronic disease conditions. The general consensus among patients who had pain reduction was overwhelmingly positive for the stem cell therapy. In addition, use of autologous cells was universally and highly acceptable in the group. These subjects, comprising of middle-aged women, reported ongoing concern that the antineuropathic medication was affecting their quality of life due to the side effects. The main side effects of concern were drowsiness, affecting work performance and social activities, and weight gain, affecting personal esteem. ${ }^{36}$ With a noticeable reduction in antineuropathic medication requirements from the stem cells, they reported a positive outlook for their future. Previously, the majority of the subjects were on a slow but escalating dose of medication, with the likelihood of increased medication requirements for the next 30-40 years of their life, assuming normal life expectancy. The current front-line drugs for the treatment of neuropathic pain exhibit minimal efficacy. ${ }^{37}$ For example, gabapentin is one of the most favorable drugs on the market. At its maximum dose $(3,600 \mathrm{mg})$, it provides pain relief to less than $60 \%$ of patients, with an NNT (numbers needed to treat) value of four. ${ }^{38}$ In our study, the near significant reduction in gabapentin use and minor reduction in amitriptyline (Figure 2) suggests that stem cells may exhibit biological priority in recovering myelinated fibers over unmyelinated fibers. This would have potentially important implications if further studies can confirm these initial findings and may possibly explain the unusual clinical history of patients with trigeminal neuralgia. Typically, the sharp lancinating pain form of classical neuralgia has pain remission episodes. On the other hand, patients with the constant burning properties of atypical trigeminal neuralgia rarely undergo periods of remission. ${ }^{39}$ Further studies of multiple dose strategies and blinded controlled trials are required and mandatory to establish a higher level of scientific evidence. This initial study showed a therapeutic response in the majority of subjects with neuropathic pain and suggests that autologous adipose stem cell therapy is a safe and efficacious procedure.

\section{Conclusion}

In this preliminary proof-of-concept study, the administration of a mixed population of adipose-derived cells, including
MSCs, was shown to be able to attenuate orofacial neuropathic pain symptoms in a diverse range of patients considering variables of age and the established duration of the pain state. The positive efficacy and safety results (7/9 patients and 9/9 patients, respectively) of this study mandates further clinical evaluation of neuropathic pain in an RCT. Moreover, the orofacial region is a likely investigative area for other neuronal degenerative conditions to assess stem cell intervention due to well-known diagnostic states and well-defined anatomical landmarks to site stem cell injections. This would include paresthesia from oral surgery $(\sim 1 \%$ of dental patients incur permanent lip/tongue numbness from third molar surgery) and facial nerve paresis. Several variables in the procedure require further investigation, including multiple stem cell injections over several months to enhance the therapeutic benefit. This report demonstrated a good patient response to a single dose of adipose-derived stem cells. Multiple dosage strategies have been demonstrated to prolong the therapeutic effect longer than what is seen with a single dosage treatment. ${ }^{16,24,40}$ Furthermore, there is an increasing number and accessibility of commercial stem cell banking facilities for cellular storage to enable multiple SVF administrations. One further variable is the critical number of stem cells required to produce a measurable change in pain relief. The authors have included the data on total cell numbers and cell viability for comparison in future studies.

\section{Acknowledgments}

The authors acknowledge the contribution of Juliana Leal from Regeneus Ltd for laboratory expertise in the processing of the lipoaspirate.

\section{Disclosure}

ER Vickers declares no financial interest. E Karsten and R Lilischkis are employees of Regeneus Ltd. J Flood holds company shares in Regeneus Ltd.

\section{References}

1. Bouhassira D, Lantéri-Minet M, Attal N, Laurent B, Touboul C. Prevalence of chronic pain with neuropathic characteristics in the general population. Pain. 2008;136(3):380-387.

2. Dieleman JP, Kerklaan J, Huygen F, Bouma PAD, Sturkenboom MC. Incidence rates and treatment of neuropathic pain conditions in the general population. Pain. 2008;137(3):681-688.

3. Bouhassira D, Attal N, Alchaar H, et al. Comparison of pain syndromes associated with nervous or somatic lesions and development of a new neuropathic pain diagnostic questionnaire (DN4). Pain. 2005;114(1-2):29-36.

4. Masson E, Hunt L, Gem J, Boulton A. A novel approach to the diagnosis and assessment of symptomatic diabetic neuropathy. Pain. 1989;38(1):25-28.

5. Zimmermann M. Pathobiology of neuropathic pain. Eur J Pharmacol. 2001;429(1-3):23-37. 
6. Milligan ED, Twining C, Chacur M, et al. Spinal glia and proinflammatory cytokines mediate mirror-image neuropathic pain in rats. $J$ Neurosci. 2003;23(3):1026-1040.

7. Okamoto K, Martin D, Schmelzer J, Mitsui Y, Low P. Pro- and anti-inflammatory cytokine gene expression in rat sciatic nerve chronic constriction injury model of neuropathic pain. Exp Neurol. 2001;169(2):386-391.

8. Vallejo R, Tilley DM, Vogel L, Benyamin R. The role of glia and the immune system in the development and maintenance of neuropathic pain. Pain Pract. 2010;10(3):167-184.

9. Gilron I, Watson CPN, Cahill CM, Moulin DE. Neuropathic pain: a practical guide for the clinician. Can Med Assoc J. 2006;175(3):265-275.

10. Backonja M, Galer BS. Pain assessment and evaluation of patients who have neuropathic pain. Neurol Clin. 1998;16(4):775-790.

11. Sindrup S, Jensen T. Efficacy of pharmacological treatments of neuropathic pain: an update and effect related to mechanism of drug action. Pain. 1999;83(3):389-400.

12. Mazzini L, Mareschi K, Ferrero I, et al. Mesenchymal stromal cell transplantation in amyotrophic lateral sclerosis: a long-term safety study. Cytotherapy. 2012;14(1):56-60.

13. Hare JM, Traverse JH, Henry TD, et al. A randomized, double-blind, placebo-controlled, dose-escalation study of intravenous adult human mesenchymal stem cells (prochymal) after acute myocardial infarction J Am Coll Cardiol. 2009;54(24):2277-2286.

14. De Ugarte D, Morizono K, Elbarbary A, et al. Comparison of multilineage cells from human adipose tissue and bone marrow. Cells Tissues Organs. 2003;174(3):101-109.

15. Blaber SP, Webster RA, Hill CJ, et al. Analysis of in vitro secretion profiles from adipose-derived cell populations. J Transl Med 2012;10:172.

16. Riordan NH, Ichim TE, Min W-P, et al. Non-expanded adipose stromal vascular fraction cell therapy for multiple sclerosis. J Transl Med. 2009;7:29.

17. Rehman J, Traktuev D, Li J, et al. Secretion of angiogenic and antiapoptotic factors by human adipose stromal cells. Circulation. 2004;109(10):1292-1298.

18. Premaratne GU, Ma L-P, Fujita M, et al. Stromal vascular fraction transplantation as an alternative therapy for ischemic heart failure: anti-inflammatory role. J Cardiothorac Surg. 2011;6:43.

19. Fain JN, Madan AK, Hiler ML, Cheema P, Bahouth SW. Comparison of the release of adipokines by adipose tissue, adipose tissue matrix, and adipocytes from visceral and subcutaneous abdominal adipose tissues of obese humans. Endocrinology. 2004;145(5):2273-2282.

20. Dadon-Nachum M, Sadan O, Srugo I, Melamed E, Offen D. Differentiated mesenchymal stem cells for sciatic nerve injury. Stem Cell Rev. 2011;7(3):664-671.

21. Guo W, Wang H, Zou S, et al. Bone marrow stromal cells produce long-term pain relief in rat models of persistent pain. Stem Cells. 2011;29(8):1294-1303.

22. Naruse K, Sato J, Funakubo M, et al. Transplantation of bone marrowderived mononuclear cells improves mechanical hyperalgesia, cold allodynia and nerve function in diabetic neuropathy. PLoS One. 2011;6(11):e27458.
23. Siniscalco D, Giordano C, Galderisi U, et al. Long-lasting effects of human mesenchymal stem cell systemic administration on pain-like behaviors, cellular, and biomolecular modifications in neuropathic mice. Front Integr Neurosci. 2011;5:79.

24. Sacerdote P, Niada S, Franchi S, et al. Systemic administration of human adipose-derived stem cells reverts nociceptive hypersensitivity in an experimental model of neuropathy. Stem Cells Dev. 2013;22(8):1252-1263.

25. Ichim T, Solano F, Lara F, et al. Feasibility of combination allogeneic stem cell therapy for spinal cord injury: a case report. Inter Arch Med. 2010;3:30.

26. Kishk NA, Gabr H, Hamdy S, et al. Case control series of intrathecal autologous bone marrow mesenchymal stem cell therapy for chronic spinal cord injury. Neurorehabil Neural Repair. 2010;24:702-708.

27. Gordh TE, Stubhaug A, Jensen TS, et al. Gabapentin in traumatic nerve injury pain: a randomized, double-blind, placebo-controlled, cross-over, multi-center study. Pain. 2008;138(2):255-266.

28. Hanna M, O'Brien C, Wilson M. Prolonged-release oxycodone enhances the effects of existing gabapentin therapy in painful diabetic neuropathy patients. Eur J Pain. 2008;12(6):804-813.

29. Serpell M, Group NPS. Gabapentin in neuropathic pain syndromes: a randomised, double-blind, placebo-controlled trial. Pain. 2002;99(3): $557-566$.

30. De Craen A, Tijssen J, de Gans J, Kleijnen J. Placebo effect in the acute treatment of migraine: subcutaneous placebos are better than oral placebos. J Neurol. 2000;247(3):183-188.

31. Moseley J, Wray N, Kuykendall D, Willis K, Landon G. Arthroscopic treatment of osteoarthritis of the knee: a prospective, randomized, placebo-controlled trial. Results of a pilot study. Am J Sports Med. 1996;24(1):28-34.

32. Kaptchuk T, Goldman P, Stone D, Stason W. Do medical devices have enhanced placebo effects? J Clin Epidemiol. 2000;53(8):786-792.

33. Stolzing A, Jones E, McGonagle D, Scutt A. Age-related changes in human bone marrow-derived mesenchymal stem cells: consequences for cell therapies. Mech Ageing Dev. 2008;129(3):163-173.

34. De Francesco F, Tirino V, Desiderio V, et al. Human CD34/CD90 ASCs are capable of growing as sphere clusters, producing high levels of VEGF and forming capillaries. PLoS One. 2009;4(8):e6537.

35. Ra JC, Shin IS, Kim SH, et al. Safety of intravenous infusion of human adipose tissue-derived mesenchymal stem cells in animals and humans. Stem Cells Dev. 2011;20(8):1297-1308

36. Galluzzi K. Management of neuropathic pain. J Am Osteopath Assoc. 2005;105(9 Suppl 4):S12-S19.

37. Connor A. Neuropathic pain: quality-of-life impact, costs and cost effectiveness of therapy. Pharmacoeconomics. 2009;27(2):95-112.

38. Finnerup N, Otto M, McQuay H, Jensen T, Sindrup S. Algorithm for neuropathic pain treatment: an evidence based proposal. Pain. 2005;118(3):289-305.

39. Boocock H, Vickers ER. Trigeminal neuralgia and its atypical variant Mod Med. 2009; 10:14-22.

40. Franchi S, Valsecchi AE, Borsani E, et al. Intravenous neural stem cells abolish nociceptive hypersensitivity and trigger nerve regeneration in experimental neuropathy. Pain. 2012;153(4):850-861.
Journal of Pain Research

\section{Publish your work in this journal}

The Journal of Pain Research is an international, peer-reviewed, open access, online journal that welcomes laboratory and clinical findings in the fields of pain research and the prevention and management of pain. Original research, reviews, symposium reports, hypothesis formation and commentaries are all considered for publication.

\section{Dovepress}

The manuscript management system is completely online and includes a very quick and fair peer-review system, which is all easy to use. Visit http://www.dovepress.com/testimonials.php to read real quotes from published authors. 